
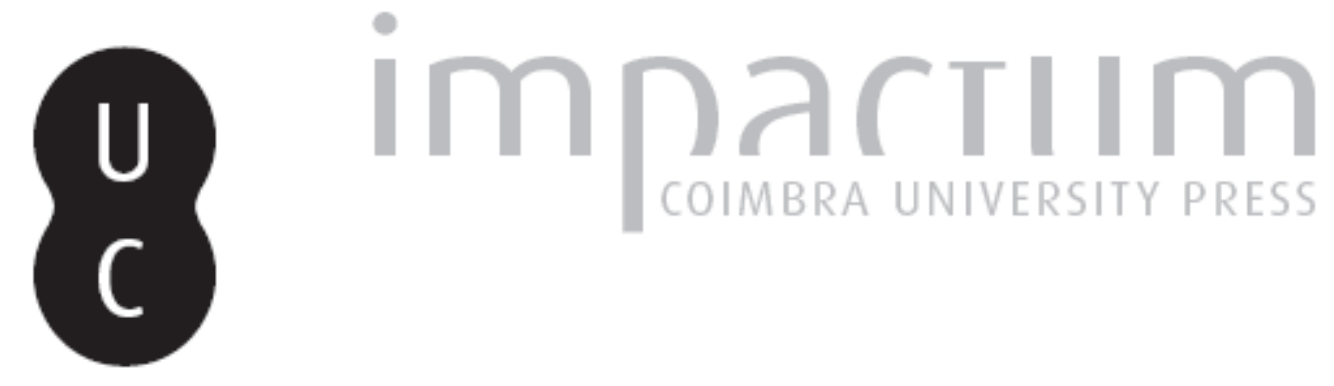

\title{
Padrões e sazonalidade do enfarte agudo do miocárdio em Portugal
}

Autor(es): $\quad$ Almendra, Ricardo; Santana, Paula; Vasconcelos, João; Freire, Elisabete

Publicado por: Faculdade de Letras da Universidade de Coimbra, Departamento de Geografia

URL

persistente:

URI:http://hdl.handle.net/10316.2/30230

DOI:

DOI:http://dx.doi.org/10.14195/0871-1623_32_22

Accessed : $\quad$ 26-Apr-2023 10:20:48

A navegação consulta e descarregamento dos títulos inseridos nas Bibliotecas Digitais UC Digitalis, UC Pombalina e UC Impactum, pressupõem a aceitação plena e sem reservas dos Termos e Condições de Uso destas Bibliotecas Digitais, disponíveis em https://digitalis.uc.pt/pt-pt/termos.

Conforme exposto nos referidos Termos e Condições de Uso, o descarregamento de títulos de acesso restrito requer uma licença válida de autorização devendo o utilizador aceder ao(s) documento(s) a partir de um endereço de IP da instituição detentora da supramencionada licença.

Ao utilizador é apenas permitido o descarregamento para uso pessoal, pelo que o emprego do(s) título(s) descarregado(s) para outro fim, designadamente comercial, carece de autorização do respetivo autor ou editor da obra.

Na medida em que todas as obras da UC Digitalis se encontram protegidas pelo Código do Direito de Autor e Direitos Conexos e demais legislação aplicável, toda a cópia, parcial ou total, deste documento, nos casos em que é legalmente admitida, deverá conter ou fazer-se acompanhar por este aviso.

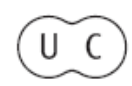




\title{
Padrões e sazonalidade do enfarte agudo do miocárdio em Portugal
}

\author{
Ricardo Almendra \\ Faculdade de Arquitetura da UTL. \\ ricardoalmendra85@gmail.com
}

\section{Paula Santana}

Departamento de Geografia e Centro de Estudos de Geografia e Ordenamento do Território (CEGOT). Faculdade de Letras da Universidade de Coimbra. paulasantana.coimbra@gmail.com)

\section{João Vasconcelos}

Instituto Politécnico de Leiria.

j.vasconcelos@ipleiria.pt)

\section{Elisabete Freire}

Faculdade de Arquitetura da UTL.

efreire@fa.utl.pt

\section{Resumo:}

Apesar do decréscimo verificado nos últimos anos, a mortalidade por doença cardiovascular em Portugal continua a ser a principal causa de morte e de internamento hospitalar. Esta patologia apresenta uma forte sazonalidade, verificando-se a concentração de óbitos e de internamentos durante os meses de inverno

A análise dos padrões e da sazonalidade do enfarte agudo do miocárdio possibilita a reconhecimento de populações em risco e a identificação de situações em que é necessário intervir.

Foi observada uma distribuição espacial contrastada no risco de internamento durante os meses de inverno, tendo sido identificado o sul e o interior do país como áreas onde o problema é mais evidente.

Palavras-chave: Doenças cardiovasculares. Enfarte agudo do miocárdio. Sazonalidade. Risco de internamento.

\section{Résumé:}

Tendances et saisonnalité de l'infarctus du myocarde au Portugal

Malgré la baisse observée pendant ces dernières années, la mortalité par maladies cardio-vasculaires, au Portugal, reste la principale cause de décès et d'hospitalisation. Cette maladie a une forte saisonnalité, vérifier la concentration des décès et des hospitalisations au cours des mois d'hiver

L'analyse de modèles et de la saisonnalité de l'infarctus du myocarde aigu permet la reconnaissance des populations à risque et d'identifier les situations où une intervention est nécessaire.

Il existe des différences claires dans la distribution spatiale du risque d'hospitalisation au cours des mois d'hiver. Il est dans le sud et l'intérieur du pays que le problème est plus évident.

Mots-clés: Maladies cardio-vasculaires. Infarctus du myocarde. La saisonnalité. Risque d’hospitalisation.

\section{Abstract:}

Patterns and seasonality of acute myocardial infarction in portugal

Though, in recent years, there has been a decrease on cardiovascular mortality, in Portugal, this is still the leading cause of death and of hospitalization. This disease has a clear seasonality with a peak of both mortality and hospital admissions during the winter months.

The analysis of the acute myocardium infarction pattern and seasonality enables identification of population at risk and also the situations that is urgent to act.

There is a strong difference on the spatial distribution in the risk of hospital admission during winter months; the south and inland areas are the most vulnerable

Keywords: Cardiovascular diseases. Seasonality. Acute myocardium infarction pattern. Risk of hospitalization. 


\section{Introdução}

Em Portugal, verificou-se nos últimos anos um importante decréscimo da mortalidade por doença cardiovascular, apesar de apresentar ainda valores superiores à média da Europa dos 15 (SANTANA, 2010). A variação da mortalidade, em especial a associada às doenças cardiovasculares, apresenta padrões típicos de sazonalidade, com maior incidência durante os meses de inverno (Falagas et al., 2009). Verificam-se efeitos adversos na saúde provocados pela exposição a temperaturas abaixo do ótimo biológico em que algumas funções vitais são afetadas (FreIRE, 1996; De Lorenzo et al., 1999; WILSON et al., 2010).

No entanto, a relação entre ambiente e saúde é mais complexa e não deve ser explicada apenas através da descida da temperatura (Nogueira et al., 2004; ANDRADE et al., 2011). De fato, é nos países com invernos mais amenos que se registam maiores aumentos sazonais (Eurowinter, 1997; Nogueira et al., 2009), sendo Portugal o país da Europa com maior excesso de mortalidade no inverno (HEALY, 2003).

Ao contrário do que acontece noutros países, em Portugal a sazonalidade da mortalidade/morbilidade é menosprezada enquanto grave problema de saúde pública. Mesmo nos casos em que o problema é reconhecido pelos serviços hospitalares, este não é alvo de uma ação concertada de mitigação ou sequer de quantificação.

O principal objetivo do presente trabalho é de identificar e caracterizar os padrões de sazonalidade das doenças cardiovasculares em Portugal Continental

\section{Material e métodos}

\subsection{Origem dos dados}

A morbilidade hospitalar foi analisada através do registo de internamentos hospitalares presentes na base de dados dos Grupos de Diagnóstico Homogéneos (GDH) proveniente da Administração Central do Sistema de Saúde. Consideraram-se os episódios de internamento em hospitais públicos do SNS de residentes do Continente, cuja entrada nos serviços hospitalares se deu no quinquénio de 2003 a 2007e que foram codificados com o diagnóstico principal de Enfarte Agudo do Miocárdio (EAM) (cid 9: 410). Os episódios considerados na análise referem-se a eventos de internamento, de acordo com o local de residência dos indivíduos, e não a indivíduos que foram hospitalizados.
Os dados populacionais são provenientes do Instituto Nacional de Estatística (INE).

\subsection{Metodologia}

Para estudar a geografia da morbilidade hospitalar por EAM calcularam-se Taxas de Internamento Padronizadas $^{1}$ (TIP) segundo o método de padronização directo e utilizando a população padrão europeia. A TIP apresenta-se como uma taxa bruta de internamentos que é ajustada considerando as diferenças existentes na estrutura etária da população através de uma população padrão. Esta padronização permite realizar comparações mais correctas entre territórios, principalmente quando as estruturas populacionais são díspares (DGS, 2006).

De modo a identificar territórios com elevado risco de internamento calcularam-se Razões Padronizadas de Internamento ${ }^{2}$ (RPI), sendo possível comparar as diversas regiões com o Continente. O cálculo das RPI faz-se em três passos: determinam-se, para o Continente, as taxas de internamento para cada grupo etário, que serão as taxas de referência; estimam-se os internamentos esperados para cada grupo de idades e para cada NUT tendo em conta a taxa de referência apurada; determinam-se as RPI através da relação entre os internamentos esperados e os observados (Santana, 2005). De modo a maximizar a precisão da informação, calcularam-se intervalos de confiança a $95 \%{ }^{3}$, segundo o referido por SANTANA (2002).

Para estudar a dimensão da sazonalidade adaptou-se o Índice de Excesso de Internamentos no inverno ${ }^{4}$ (IEII) utilizado pelo UK National Statistics. Este índice mede o excesso de internamentos no inverno, comparando o número de internamentos no inverno (Novembro a Março) com o número médio de internamentos no período não-inverno (Abril a Outubro) e expressa-se em percentagem (WMPHO, 2011). Através do mesmo método é possível identificar o número de internamentos em excesso no inverno.

Aplicou-se um factor de normalização ${ }^{5}$ aos valores mensais o que permitiu obter resultados padronizados a meses de 30 dias (PINHEIRO, 1990)

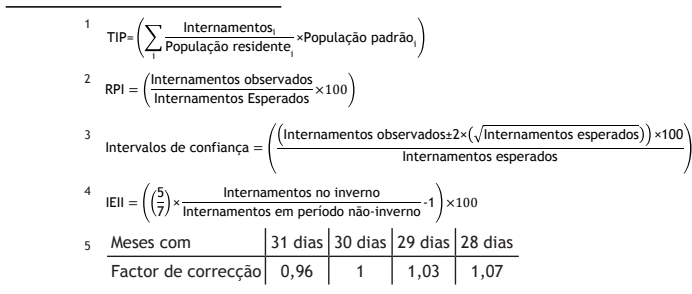




\section{Resultados e discussão}

\subsection{Padrões de internamento}

Em Portugal Continental foram identificados 60 305 episódios de internamento por EAM, no quinquénio 2003-07. O número de internamentos apresenta pequenas variações no período em estudo, sugerindo, contudo, uma tendência decrescente de 2003 a 2007 (Figura 1).

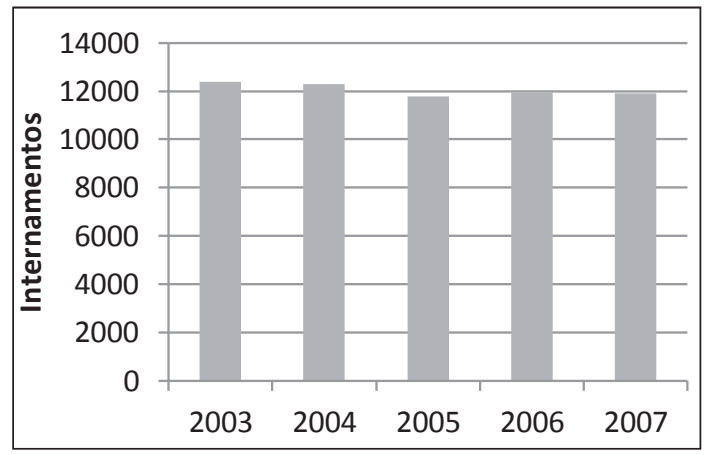

Figura 1

Internamentos por Enfarte Agudo do Miocárdio por ano

Fonte: Elaboração própria através da base de dados dos GDH (ACSS).

Verificou-se também um aumento dos internamentos por EAM com a idade; tendência que é comum nos Homens e nas Mulheres. Todavia, no sexo masculino atingem valores mais elevados mais cedo, registando 400 internamentos por 100000 habitantes na classe etária dos 65 aos 69 anos, enquanto as Mulheres apenas atingem esse valor na classe dos 80 aos 84 anos (Figura 2). Os internamentos em idades inferiores a 35 anos são muito raros, em ambos os sexos, e representam menos de $1 \%$ do total $(0,7 \%)$.

A análise da TIP revela um padrão geográfico assimétrico com grandes disparidades: Baixo Alentejo, a região com maior TIP, apresenta resultados $120 \%$ superiores ao Pinhal Interior Sul, que é a região com menor taxa. Destacam-se, ainda, as NUT do Grande Porto, Cova da Beira, Península de Setúbal e Alentejo Central com valores de internamentos muito elevados, enquanto as regiões do interior Norte e Centro apresentam os valores mais baixos, com a excepção de Cova da Beira (Figura 3).

Este padrão foi identificado em estudos prévios. Por exemplo, Freire (1996), Santana (2005), Nicolau et al (2009) e ACS (2010) AlmendRa (2011) indicam o interior Sul e o Alentejo como propensos à doença coronária, quer em internamentos quer em mortalidade.

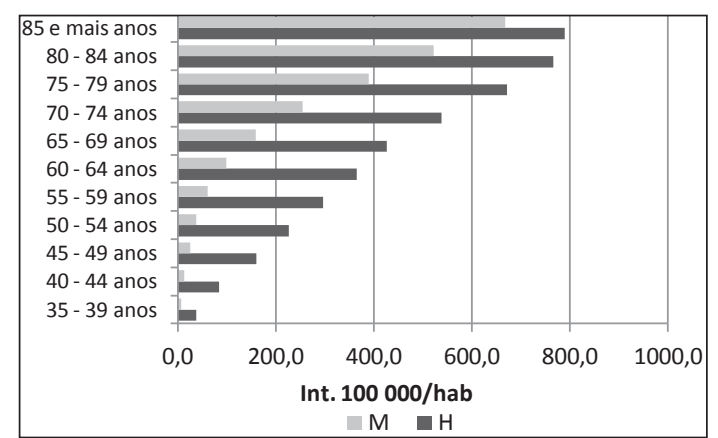

Figura 2

Taxa bruta de internamento por Enfarte Agudo do Miocárdio (2003/07) Fonte: Elaboração própria através da base de dados dos GDH (ACSS).

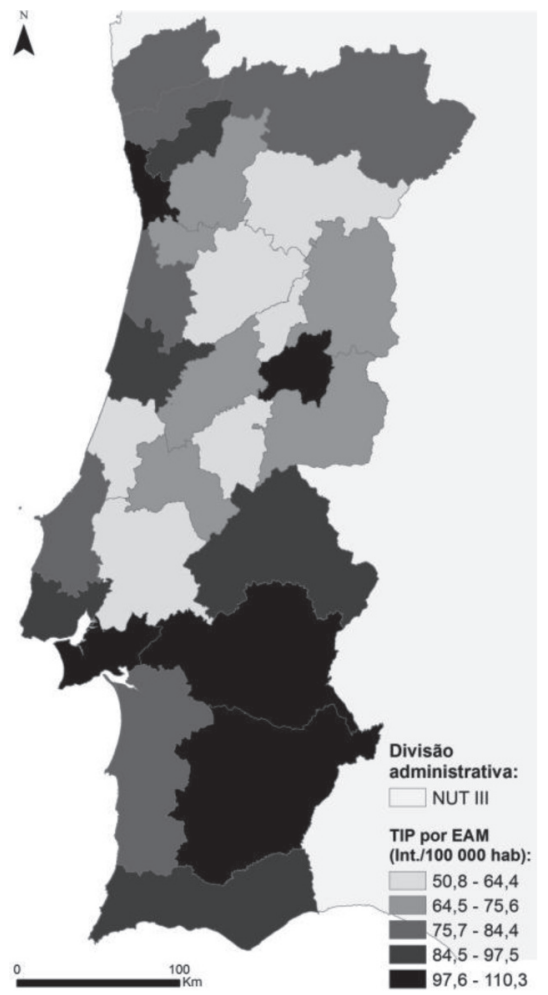

Figura 3

TIP por Enfarte Agudo do Miocárdio (2003/07)

Fonte: Elaboração própria através da base de dados dos GDH (ACSS).

\subsection{Sazonalidade}

A análise da distribuição mensal do número de internamentos por EAM permite concluir a existência de uma sazonalidade. De facto, os meses de Novembro a Março, referidos aqui como meses de inverno, apresentam um volume de internamentos superior à média mensal. 0 mês de Dezembro regista o maior número de internamentos (5696), contrastando com Julho que 
apresenta 4307 internamentos. Este padrão é semelhante na população idosa, registando-se igualmente excesso de internamentos durante o inverno (Figura 4). Ou seja, apesar do excesso de internamentos ser verificável nos dois grupos populacionais, é mais importante nos idosos; no mês de Dezembro os idosos registaram um aumento relativo de $15 \%$ em relação à média, enquanto a população total registou um aumento de $12 \%$. Nos meses de Verão, com registo de menor número de internamentos, os idosos apresentam o maior decréscimo; em Agosto verifica-se uma variação negativa de $25 \%$ em relação à média mensal na população com mais de 64 anos e de 19\% na população total (Figura 5).

Apesar da grande assimetria existente entre as regiões, identificou-se excesso de internamentos em 27 das 28 NUT, quando considerando a população total. A
Beira Interior Norte, é a região com sazonalidade mais vincada, apresentando $43 \%$ de excesso de internamentos durante o inverno. Em sentido oposto, Minho Lima é a região que apresenta menores valores, com $6,4 \%$. Uma referência a Pinhal Interior Sul, que não apresenta excesso (Figura 6).

É possível identificar um padrão que corresponde a valores superiores a $40 \%$, com destaque para as subregiões do interior Centro e Sul (Beira Interior Norte, o Alto Alentejo e Alentejo Central). Os valores mais baixos (inferiores a 10\%) são observados no Minho Lima, Lezíria do Tejo e Oeste.

Analisando a população idosa verifica-se, desde logo, a existência de excesso de internamentos em todas as regiões. O Alto Alentejo e o Alentejo Central (com, respectivamente, $48 \%$ e $41 \%$ ) correspondem às
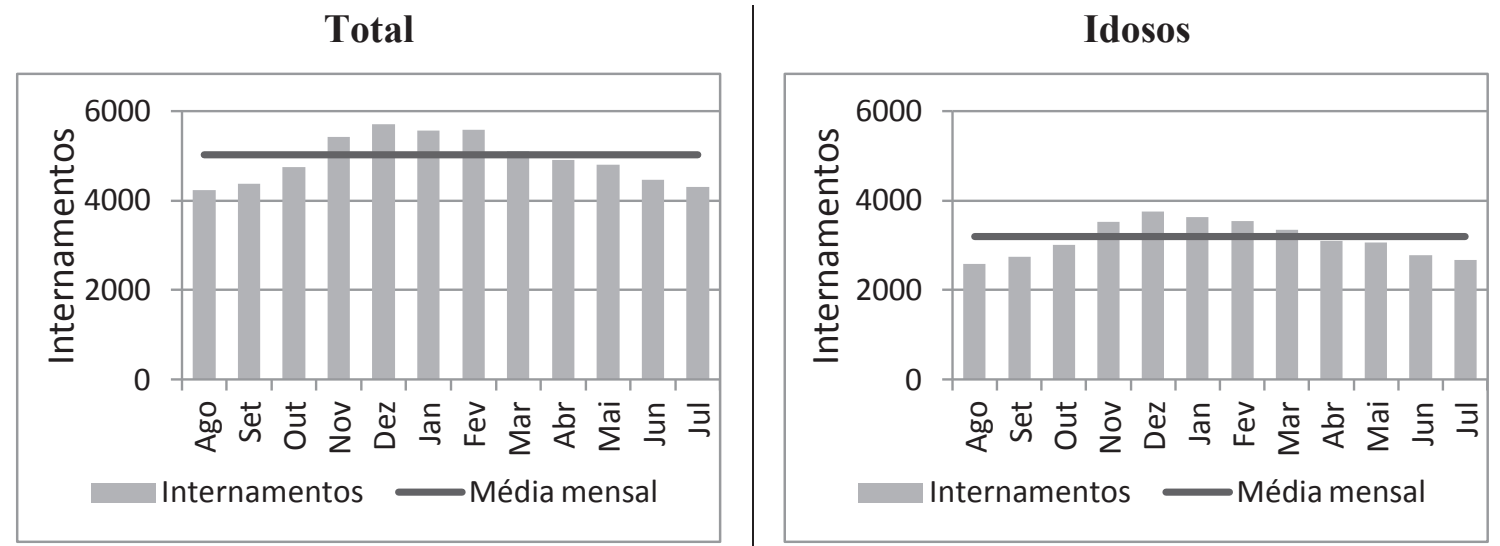

Figura 4

Internamentos por Enfarte Agudo do Miocárdio por mês (2003/07), na população total e de idosos Fonte: Elaboração própria através da base de dados dos GDH (ACSS)

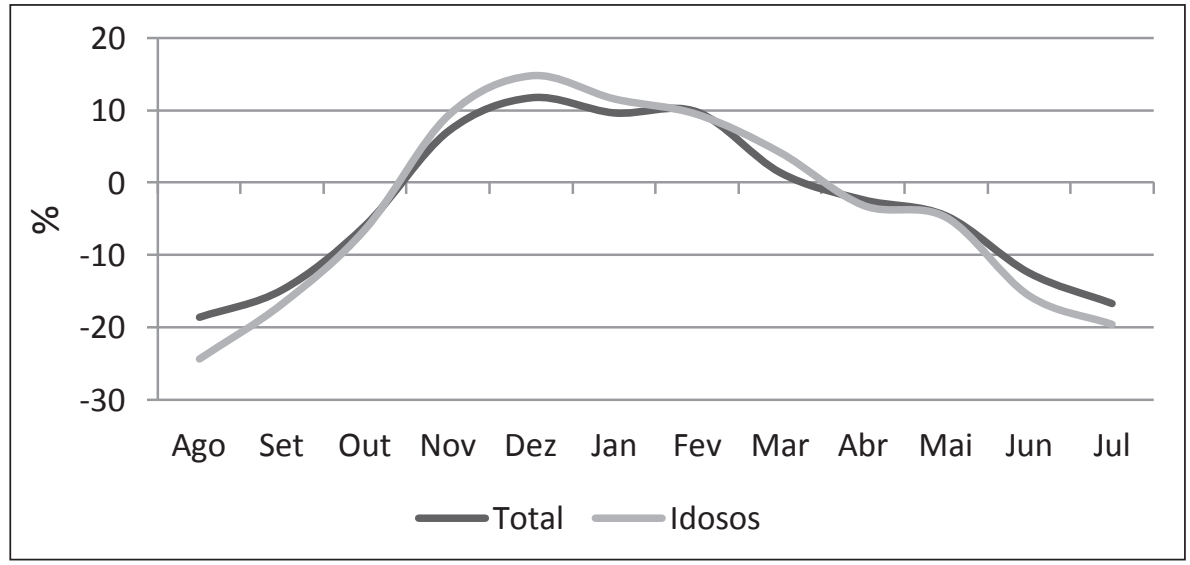

Figura 5

Variação relativa do número de internamentos por Enfarte Agudo do Miocárdio, ao longo do ano (2003-07) Fonte: Elaboração própria através da base de dados dos GDH (ACSS) 
sub-regiões com maior excesso de internamentos, por outro lado Pinhal Interior Sul, Alto Trás-os-Montes e Douro apresentam valores inferiores a $10 \%$.

A sazonalidade, expressa pelo excesso de mortalidade no inverno, é referida na literatura científica de medicina desde há 150 anos (Healy, 2003) e, por isso, as reações corporais provocadas pela exposição ao frio encontram-se amplamente discutidas. A literatura revela, por exemplo, o aumento da frequência cardíaca, diminuição da pulsação e da frequência respiratória, que se traduz em acrescido risco de coagulação do sangue e de infeções nas vias aéreas (Freire, 1996; MAHESWARAN et al., 2004; Bhaskaran et al., 2010).

Nas últimas décadas a sazonalidade tem vindo a diminuir gradualmente (Kunst et al., 1994; CARSON et al., 2006), no entanto continua a ser bastante mais elevada em países como Portugal, Espanha, Irlanda, Inglaterra e País de Gales (Lawlor et al., 2000; Healy, 2003) do que noutros países europeus com clima semelhante, revelando-se mesmo como uma preocupação em termos de saúde pública nalguns destes países. 0 facto do excesso de mortalidade no inverno ser mais pronunciado em climas amenos do que em climas frios, sugere que a

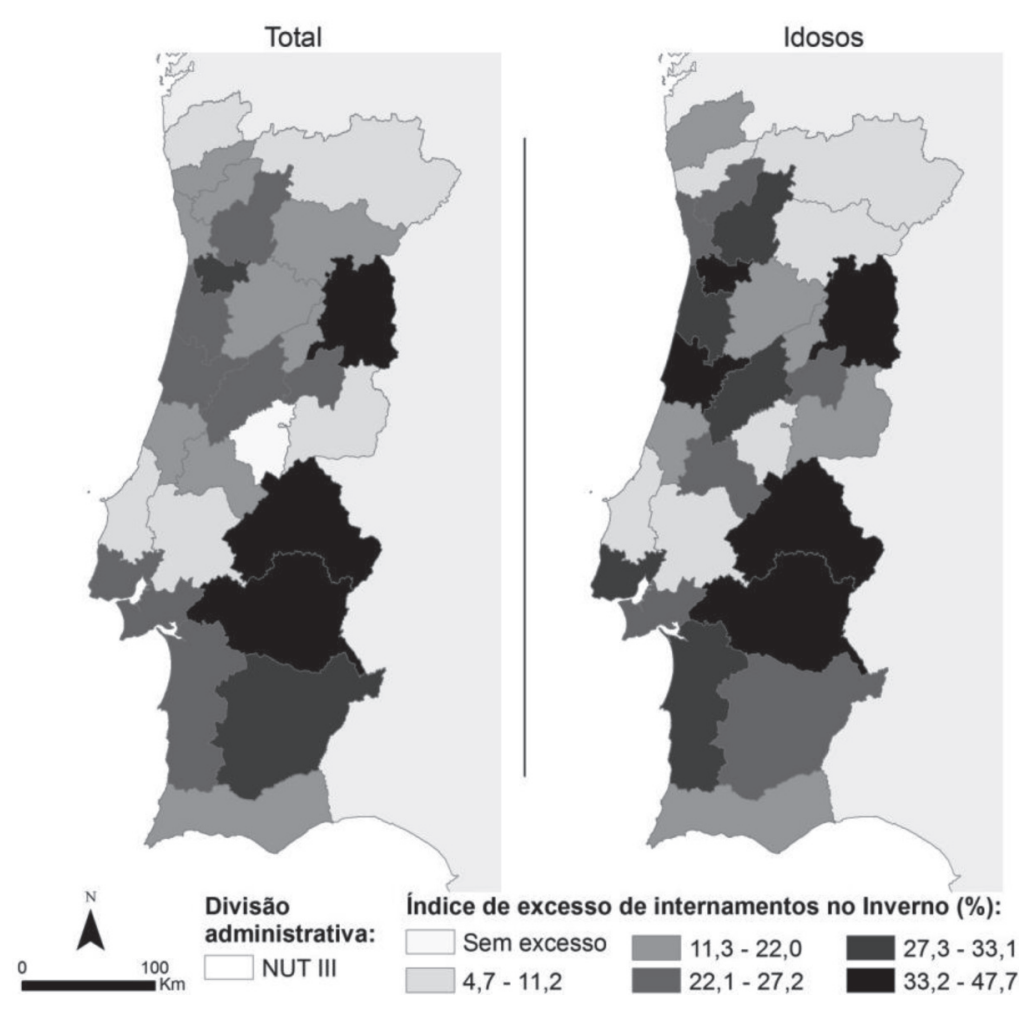

Figura 6

Índice de excesso de internamentos por Enfarte Agudo do Miocárdio no inverno (2003/07), na população total e de idosos

Fonte: Elaboração própria através da base de dados dos GDH (ACSS) sua explicação está mais associada a outros fatores que não apenas ao da temperatura. Os fatores explicativos mais referidos são comportamentais (EUROWINTER, 1997; MCKeE et al., 1998; Goodwin, 2000) e de qualidade da habitação (Healy, 2003; HaJat et al., 2007; El Ansari E ElSILIMY, 2008).

\subsection{Risco de internamento}

A análise do risco de internamento revela que 7 sub-regiões apresentam resultados significativamente superiores ao Continente (Algarve, Baixo Alentejo, Alentejo Central, Península de Setúbal, Grande Lisboa, Grande Porto e Cova da Beira). O padrão parece evidenciar as sub-regiões do Sul, com especial destaque o interior Sul, como particularmente propensas ao EAM. Por outro lado, a quase totalidade das NUT do Norte e Centro consubstanciam-se como regiões de baixo risco de internamento (Figura 7).

Analisando o risco de internamento em excesso no inverno verifica-se um padrão relativamente semeIhante: Grande Porto, Grande Lisboa, Alentejo Central e Baixo Alentejo surgem, igualmente, como regiões de elevado risco de internamento. As NUT do Cávado, 
Beira Interior Norte, Baixo Mondego e Alto Alentejo, que não apresentavam um elevado risco de internamento, registam uma sazonalidade mais marcada que a do Continente.

A incidência do EAM é frequentemente associada a determinantes de natureza biológica (hipertensão, colesterol elevado, sexo, idade) e comportamental (consumo de tabaco, dieta, sedentarismo, consumo de álcool), entre outros (Goodwin, 2000; SANTANA, 2005). Os padrões da distribuição territorial da doença cardiovascular podem em parte ser explicados pela própria geografia desses determinantes.

Por outro lado, para explicar a distribuição desigual da doença ao longo o ano devem-se considerar determinantes de cariz ambiental como a exposição ao frio ou as condições de habitação (FreIRE, 1996; HeAly, 2003). O estudo de Healy (2003) reafirma a preocupação com o tema, por ter identificado Portugal como o país da Europa com maior percentagem de variação da mortalidade durante o inverno, seguida da Espanha e Irlanda, indicando que a causa provável para os elevados valores verificados em Portugal esteja associada às condições precárias das habitações, à falta de aque-
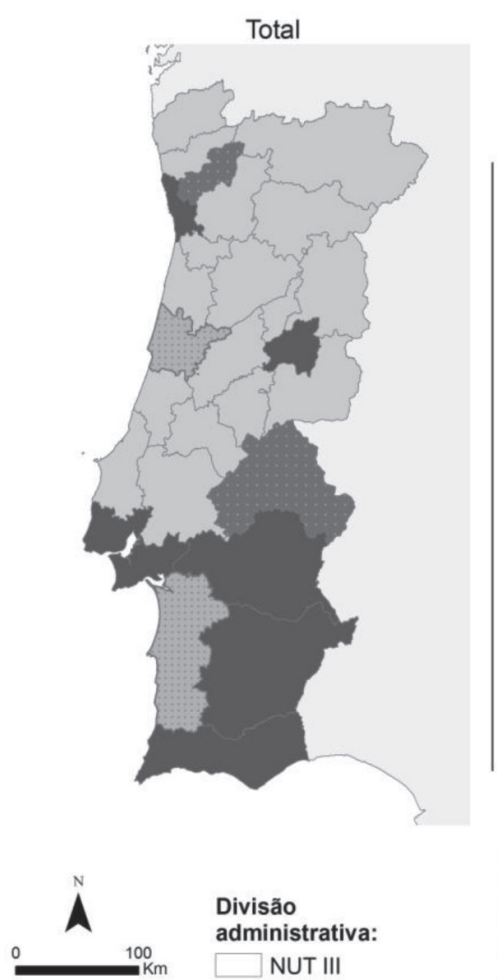

$$
\begin{aligned}
& \text { Divisão } \\
& \text { administrativa: }
\end{aligned}
$$

NUT III cimento do ar nos espaços interiores, deficiente isolamento térmico dos edifícios e, ainda, à iniquidade dos rendimentos, quando comparados com outros países da Europa.

Estas razões parecem não ser suficientes para explicar o padrão que se verifica, uma vez que as regiões do interior apresentam resultados positivos, o que poderia sugerir que as condições de habitação/edifícios são mais adequadas ou que as populações se protegem melhor. Esta é uma hipótese que merece ser estudada. A capacidade de reconhecer o perigo da exposição ao frio, nomeadamente os comportamentos de protecção que o indivíduo adopta, assumem-se como factores determinantes para explicar o excesso de internamentos no inverno e, nesse sentido, merecem ser avaliados em trabalhos futuros (Maheswaran et al., 2004; HaJat et al., 2007; VAsConcelos et al., 2011).

A explicação dos resultados proveniente da análise da morbilidade, através dos internamentos hospitalares, deve ter subjacente o possível viés da informação, resultante do desigual acesso aos serviços de saúde, com potenciais consequências no volume de internamentos por sub-região.

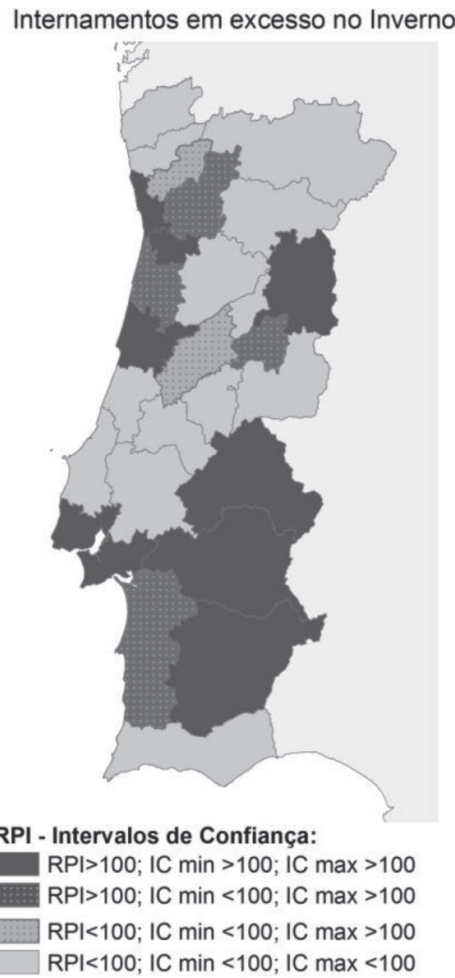

Figura 7

Razão Padronizada de Internamentos por Enfarte Agudo do Miocárdio totais e em excesso no inverno (2003-07)

Fonte: Elaboração própria através da base de dados dos GDH (ACSS) 


\section{Conclusão}

Verificou-se que a morbilidade hospitalar por EAM aumenta com a idade, sendo superior nos Homens, e tem um padrão marcadamente geográfico; as regiões do Sul, em particular do Interior Alentejano, apresentam as TIP mais elevadas; valores mais baixos verificaram-se nas regiões do Interior Norte e Centro.

Os internamentos aumentam nos meses de inverno (excesso de internamentos de Novembro a Março), verificando-se acréscimo mais relevante nos idosos. Existem, novamente, grandes disparidades geográficas: o Índice de Excesso de Internamentos no inverno varia entre 0 e 43\%, respectivamente no Pinhal Interior Sul e na Beira Interior Norte.

Verificou-se que as regiões onde se registou maior risco de internamento durante o inverno são, simultaneamente, as regiões onde se observaram as maiores taxas de incidência de EAM.

Apesar de Portugal ser dos países da Europa com maior excesso de mortalidade durante o inverno este fenómeno ainda não se encontra suficientemente estudado e há, ainda, bastantes dúvidas relativamente ao modo como interpretar e explicar alguns resultados.

\section{Bibliografia}

ACS (2010) - Atlas do Plano Nacional de Saúde. ACS, Lisboa.

ALMENDRA, Ricardo (2011) - Geografia da doença cardiovascular: Enfarte Agudo do Miocárdio - padrões e sazonalidade. Dissertação de Mestrado, Universidade de Coimbra, Coimbra.

Andrade, Henrique; Alcoforado, Maria e Oliveira, Sandra (2011) "Perception of temperature and wind by users of public outdoor spaces: relationships with weather parameters and personal characteristics". International journal of biometeorology, 55, pp. 665-680.

Bhaskaran, Krishnan; Hajat, Shakoor; Haines, Andy; HerRetT, Emily; Wilkinson, Paul e SMEETH, Liam (2010) - "Short term effects of temperature on risk of myocardial infarction in England and Wales: time series regression analysis of the Myocardial Ischaemia National Audit Project (MINAP) registry". BMJ, 341: c3823.

Carson, Claire; Hajat, Shakoor; Armstrong, Ben e Wilkinson, Paul (2006) - "Declining vulnerability to temperature-related mortality in London over the 20th century". American Journal of Epidemiology, 164/1, pp. 77-84.

De Lorenzo, Francesco; Sharma, V.; Scully, Michael e KaKkar, Vijay (1999) - "Cold adaptation and the seasonal distribution of acute myocardial infarction". Quarterly Journal of Medicine, 92/12, pp. 747-751.

DGS (2006) - Risco de Morrer em Portugal 2006. DGS, Lisboa.

El ANSARI, Walid; EL-SILIMY, Sally (2008) - "Are fuel poverty reduction schemes associated with decreased excess winter mortality in elders? A case study from London, U.K”. Chronic Illness, 4/4, pp. 289-294.

EUROWINTER (1997) - "Cold exposure and winter mortality from ischaemic heart disease, cerebrovascular disease, respiratory disease, and all causes in warm and cold regions of Europe". The Lancet, 349/9062, pp. 1341-1346.

Falagas, Matthew; Karageorgopoulos, Drosos; Moraitis, Lampros; Vouloumanou, Evridiki; Roussos, Nikolaos; Peppas, George e RAFAILIDIS, Petros (2009) - "Seasonality of mortality: the September phenomenon in Mediterranean countries". Canadian Medical Association Journal, 181/8, pp. 484486.

FreIRE, Elisabete (1996) - The comfort climatology of Portugal, a contribution to human bioclimatology. Dissertação de Doutoramento, Universidade de Londres, Londres.

Goodwin, James (2000) - "Cold stress, circulatory illness and the elderly". In: RUdGE, Janet; NicoL, Fergus (Ed.) Cutting the cost of cold: affordable warmth for healthier homes. London, E \& FN Spon, pp. 47-60.

HaJat, Shakoor; Kovats, Sari e Lachowrcz, Kate (2007) - "Heatrelated and cold-related deaths in England and Wales: who is at risk?". Occupational and Environmental Medicine, 64/2, pp. 93-100.

HeALY, John (2003) - "Excess winter mortality in Europe: a cross country analysis identifying key risk factors". Journal of epidemiology and community health, 57/10, pp. 784789.

Kunst, Anton; Groenhof, Feikje e MACKENBACH, Johan (1994) - "The association between two windchill indices and daily mortality variation in The Netherlands". American Journal of Public Health, 84/11, pp. 1738-1742.

Lawlor, Deborah; Harvey, Daniel e Dews, Howard (2000) "Investigation of the association between excess winter mortality and socio-economic deprivation". Journal of Public Health Medicine, 22/2, pp. 176-181.

Maheswaran, Ravi; Chan, D.; Fryers, Paul; McManus, Chris e McCabe, Heather (2004) - "Socio-economic deprivation and excess winter mortality and emergency hospital admissions in the South Yorkshire Coalfields Health Action Zone, UK". Public Health, 118/3, pp. 167-176.

McKee, Martin; Sanderson, Colin; Chenet, Laurent; Vassin, Sergei e SHKolnikov, Vladimir (1998) - "Seasonal variation in mortality in Moscow". Journal of Public Health Medicine, 20/3, 268-274.

Nicolau, Rita; Machado, Ausenda; Falcão, José e Lira, Mafalda (2009) - Distribuição dos Internamentos Hospitalares 
em Portugal Continental: Agregação Geográfica e Determinantes. INSA, Lisboa.

Noguelra, Paulo; Paixão, Elonora e Rodrigues, Emanuel (2009) Sazonalidade e Periodicidades do Internamento Hospitalar em Portugal Continental - 1998 a 2003. INSA, Lisboa.

Pinheiro, Carlos (1990) - "Um frio de morrer ou a variação da mortalidade e clima nos distritos de Viana do Castelo e de Faro". Arquivos do Instituto Nacional de Saúde, 15, pp. 61-112.

Santana, Paula (2002) - "A Mortalidade "evitável" em Portugal Continental, 1989 a 1993". Revista de Estudos Demográficos, 32, pp. 107-145.

Santana, Paula (2005) - Geografias da saúde e do desenvolvimento. Evolução e tendências em Portugal. Almedina, Coimbra.
Santana, Paula (2010) - "Os Ganhos em Saúde e no Acesso aos Serviços de Saúde", in: Simões, J. (coord.), Trinta anos do Serviço Nacional de Saúde. Um percurso comentado. Edição Gulbenkian /APES (p. 21 - 81).

VAsconcelos, João; Freire, Elisabete; MoRals, João; MACHADo, João e Santana, Paula (2011) - "The health impacts of poor housing conditions and thermal discomfort". Procedia Environmental Sciences, 4, pp. 158-164.

Wilson, Thad; GaO, Zhaohui; Hess, Kari e Monahan, Kevin (2010) "Effect of aging on cardiac function during cold stress in humans". American Journal of Physiology - Regulatory, Integrative and Comparative Physiology, 298/6, pp. 1627-1633.

WMPHO (2011) - Excess Winter Deaths Atlas: User guide. West Midlands Public Health Observatory, Birmingham. 